\title{
TELEVISION AND FILM UNIT AT THE LONDON ZOO
}

\section{$\mathrm{T}$} HE Zoological Society of London, its Gardens at Regent's Park and its Park at Whipsnade have always enjoyed a great deal of newspaper and radio publicity. With the advent of the Independent Television Authority (ITA) several programmeproducing companies have been formed, and some of them have asked the Zoological Society of London to grant them television rights. The Society takes the view, however, that it would be wrong to grant exclusive rights to any one company, and that it would be equally impossible to have numerous companies working on the Society's premises, perhaps even at the same time, and disturbing its work. Exclusive rights cannot be granted nor can a com. pletely non-exclusive arrangement be contemplated. The Society has therefore decided to make the best possible zoological material available and at the same time to retain control of the Society's interests in television programmes and films, and to ensure that no responsible organization is denied the opportunity of broadcasting the programmes of its work. This will best fulfil the Society's policy of presenting its work to the public.

Of the several approaches made to the Society, all of which were carefully considered, that made by Granada was by far the most attractive and practicable. Granada proposed that the Society, in conjunction with Granada, should establish a permanent resident Television and Film Unit to produce programmes for television, for the einema, and for more specific educational and scientific purposes. The Unit will be under the direction of a universitytrained zoologist and will have at least two cameramen, perhaps more, preferably people with some scientific interest in zoology. It will cover all the work of the Society at Regent's Park, Whipsnade, on expeditions abroad or wherever it may be necessary to go for special material. Its first function will be to provide the Society with documentary material, and it will be able to undertake long-term projects which may need a considerable period of time for their completion.
The Society looks to television for something more than revenue alone. It is the medium that can best project the Society's work and encourage the primary object of the Society's Royal Charter, granted in 1829, which is "the advancement of Zoology and Animal Physiology and the introduction of new and curious subjects of the Animal Kingdom" on a scale far beyond the possibilities previously available. The work of the Unit will fulfil these objects laid down in the Charter, and while it will not suffer from any lack of scientific integrity it will have a wide appeal to the public. The use of the new medium will do much more than exploit the easy entertainment value of animals; the possibilities for such a Unit are enormous and have never been grasped by any organization before.

In addition to its long term projects and quarterly schedules of work, the Unit will keep a daily diary in picture and sound of events of special interest, now arrivals and so on, thus building up an extensive film and sound library for use in making programmes, educational and research films. Documentary matter needing the slow accumulation of material over months or even years will be recorded, and zoological expeditions to all parts of the world will also be an important part of the work of the Unit. Techniques to be used will include photomicrography, slow-motion cinematography, infra-red lighting and the 'hidden eye' to observe animal behaviour continuously over long periods.

The films will be available for production of television programmes and also for exhibition in cinemas, universities and schools. In addition, live broadeasts will be regularly produced. Granada will in general have first option; but the television programmes will be available to other users. Granada will distribute the programmes and films.

Under this arrangement the B.B.C., with which the Society has co-operated fully for many years, will be able to broadcast programmes of zoological interest in association with the new Unit.

\section{SMOKE ABATEMENT IN BRITAIN}

\section{CONFERENCE IN BOURNEMOUTH}

$\mathrm{T}$ IHE National Smoke Abatement Society may take credit for having provided for many years a platform on which discussion of the problems of smoke abatement have been given full scope. All elements of the community have in their turn been given their say. From year to year there have been heard the oft-repeated claims of the crusaders for cleaner air. For quite a few years careful research work and surveys of atmospheric pollution have been carried out by the Atmospheric Pollution Research Committee, and a conference of representatives of local authorities has been held under the regis of the Department of Scientific and Industrial Research. One must not forget, too, the contributions to technical development, which have a bearing on these problems, made by a large body of scientists and engineers in fuel technology and resulting in a knowledge of how to burn fuel smokelessly. The Society's platform has again in these fields been the means of publicizing from time to time the painstaking efforts in technical development that must provide the basis of any really effective action in smoke abatement.

In this critical year of the Society's history, the Government's Clean Air Bill is likely to be debated. Accordingly, the proceedings of this year's annual conference of the Society, held in Bournemouth during September 28-30, are of particular interest. In keeping with past traditions, all aspects of the thorny problems to be faced have in some measure received attention, and opinions have been voiced which ought to reach the ears of the legislators. In his presidential address to the Society, Sir Ernest Smith rightly stressed the influence, in bringing about this situation, of the informed body of opinion into 
which there have been injected a large variety of ideas and much practical understending by the activities of the Society. This is not to disregard the contributions made by that other large but silent body of expert opinion containing the practitioners of the technologies involved, who for upwards of a generation have provided a technical basis upon which smokeless combustion practice may be achieved. Without the 'know how' to burn fuel smokelessly, legislative action would be ineffective. The need at the present time is the problem of effective application of this existing knowledge.

The conference this year has provided interesting descriptions of recent progress in domestic heating by centralized methods, in particular of the Westminster City Council's district-heating scheme, considered from the financial, engineering and operational aspects. The scheme shows a saving of coal as well as an overall economy, a reduction of atmospheric pollution and improvement in domestic amenities.

Floor warming was an ancient practice known to communities as wide apart as the early Koreans and the Romans. To-day, with the application of electrical heating, the principle of thermal storage may be applied, the electricity being supplied to concrete flooring in 'off-peak' periods. Gradually, but surely, the hardy and reluctant dwellers of the British Isles are succumbing to the lure of the use of partial central heating, an interesting new feature of this practice being the application of forced circulation of hot water in small pipes - a result of research by the British Coal Utilization Research Association. All the heat from the boiler is kept within the living space of the house and is localized mainly in the radiators as the piping is so small. Another interesting article explains how the stuffiness of ducted hot air, another practice emanating from the Romans, may be safeguarded by suitable control of the movement of the air. The above are merely a selection of the many interesting points emerging from the technical discussions of the conference.

On the issues involved in the projected Clean Air Bill, opinions were many and comprehensive, ranging from contributions by the representatives of local authorities to several informative explanations of the purport of the Beaver Report by members of the Committee. These make much more intelligible reading than does the draft of the Clean Air Bill itself. Proverbially, the draft of a Parliamentary Bill in any case provides a form of mental persecution for the layman. The contributions to the Bournemouth conference give at least a clear indication of the intentions of the Report, and the reactions of much representative opinion from the local author. ities. The views of the ordinary man and of the industrialist were unfortunately not heard; this is to be regretted, since they may be regarded in some respects as the victims for the sacrifice.

The objectives of the conference were, as would be expected, altruistic and worthy. The factors of practicability of some of the intentions may be quite another matter, about which, it is to be hoped, something may at last be heard in the debates in Parlia. ment, if the victims have not become mesmerized. by the certainty of a kill. Nevertheless, it must be agreed that now there looms before us some prospect of wise action which may contribute materially to a cleaner Britain that is so much desired by all. How much cause for thanksgiving may emerge in next year's conference.

R. J. SARJANT

\section{CAMBRIDGE ASTRONOMICAL SYMPOSIUM}

THE symposium on "Astronomical Seeing", held at the Observatories, University of Cambridge, during September 12-14, was timed to follow the General Assembly of the International Astronomical Union at Dublin, and thus to take advantage of the presence in Europe at this time of astronomers from all over the world. Its object was to provide a forum for the exchange of ideas and information relating to the various aspects of the subject. These aspects include the accumulated knowledge of visual observers; the extensive ad hoc instrumental studies that have been made more recently, especially in the United States; the theory of scintillation, developed mainly with particular reference to radio problems ; and meteorological information about inhomogeneity and turbulence in the atmosphere. The aim was to provide time in the programme for sufficient discussion, so that progress could be made towards the resolution of real differences.

Dr. J. S. Hall (U.S. Naval Observatory, Washington, D.C.) described experiments in which correlations had been found between intensity scintillation in certain frequency-ranges and the speed of the upper winds. Lamps attached to balloons showed less scintillation even at a height of $30,000 \mathrm{ft}$. than did stars observed at the same time ; but it is probably unsafe, he said, to conclude from the experiments so far made that appreciable seeing effects come from greater heights. Dr. Hall showed a film of shadow bands crossing the objective of the Washington 40-in. telescope, and of extra-focal Hartmann patterns. Dr. P. B. Fellgett (Cambridge Observatories) reviewed the physical theory of scintillation. Plane wavefronts of starlight are diffracted by irregularities in the refractive index of the air, and the diffracted beams interfere to give phase- or amplitude-scintillation according to their mutual phases, which depend on the distance from the irregularities. Brightness-scintillation is usually negligible just below the irregularities, and increases with distance, while phase scintillation decreases slightly. The complex autocorrelation function of the amplitude across the mean direction of travel remains constant; and the mean intensity distribution in a telescopic image is also independent of distance from the source of disturbance. The spatial frequencies in the scintillation differ in general from those in the irregularities of refractive index. Dr. A. Hewish (Cavendish Laboratory) presented quantitative results for some chosen cases. $\mathrm{He}$ showed calculations and slides of the detailed development of brightness changes beyond sinusoidal phase-changing layers of various amplitudes; of the interchange between brightness changes and phase-scintillation; and of the corre. sponding autocorrelation functions of complex amplitude, showing the dominance of harmonics of the layer-frequency when the phase change is large. The corresponding statistical developments were shown for a layer having a Gaussian autocorrelation function of refractive index.

Dr. G. Keller (Perkins Observatory, Ohio) gave a detailed account of work at the Perkins Observatory. Observations have been made of the temporal powerspectra of brightness changes for various telescope apertures; the correlation of scintillation in certain frequency-ranges with upper-wind speed; correlation of upper-wind direction with the dependence of 